\title{
The ophthalmic health and refractive state of working dogs in South Brazil
}

\author{
Juliana Kravetz de Oliveira ${ }^{1}$, Mariza Bortolini1 ${ }^{1}$, Melissa Schaller ${ }^{1}$, Rafaela Kava Schuchmann¹, Bret A. Moore ${ }^{2}$ \\ and Fabiano Montiani-Ferreira ${ }^{1 *}$ \\ ${ }^{1}$ Department of Veterinary Medicine, Federal University of Paraná, Curitiba, Paraná, Brazil \\ ${ }^{2}$ Veterinary Medical Teaching Hospital, University of California, Davis, CA 95616, USA
}

\begin{abstract}
Background: Working dogs, such as police dogs and guide dogs, have important roles in the contemporary society by performing specific and demanding jobs. Ocular health and the maintenance of good visual acuity are imperative to strong work performance and thus human safety.

Aim: The aim of this study was to assess ophthalmic abnormalities and refractive errors in police and guide dogs in Brazil.

Methods: A total of 71 dogs (141 eyes) were evaluated. Ten were guide dogs and 61 were police dogs. The work performance was assessed by a questionnaire to each dog's handler/owner. All the dogs underwent a complete ocular examination, and abnormalities were classified by condition, if they were active or inactive and if they were located within the visual axis. In addition, 62 dogs were evaluated by streak retinoscopy for refractive errors.

Results: Ophthalmic abnormalities were detected in 38 (54\%) dogs, of which 23 were considered inherited, 25 were considered active, and 10 were located within the visual axis. Incipient cataracts were the most prevalent abnormality. No guide dog had an abnormality within the visual axis. The most common refractive error was myopia with the median and interquartile range of $-0.75 \pm 0.75$ diopters; among these, police dogs had $-1.0 \pm 0.5$ diopters, whereas guide dogs $+0.38 \pm 0.75$ diopters. Police dogs tended to be slightly myopic and guide dogs were emmetropic.

Conclusion: Despite finding a considerable number of ophthalmic abnormalities and refractive error, work performance was good with no signs of visual impairment in any dog. Regular ophthalmic examinations are advised for working dogs, and an exclusion of severely affected dogs from breeding programs is recommended.
\end{abstract}

Keywords: Cataract, Emmetropia, Guide dogs, Myopia, Police dogs.

\section{Introduction}

For centuries, dogs have worked alongside humans in a variety of roles (Sloane, 1955; Handy et al., 1961; Fishman, 2003; Dever, 2015). Two subgroups of specialized working dogs have more recently become important in very specific work roles: police dogs and service dogs. Police dogs are chosen and trained to perform a diverse number of activities based on their primary skill or ability, including protective guard in dangerous situation, such as criminal confrontation and olfactory detection of narcotics, explosives, and/or people (Sloane, 1955; Handy et al., 1961; Gazit and Terkel, 2003). Service dogs, however, primarily assist people with specific needs or disabilities, including serving as guide dogs for the blind or severely visually impaired (Riederle, 1999; Fishman, 2003; Craigon et al., 2017).

The working dogs are costly to select and train, and thus, maintaining them at a high level of performance as long as possible is desired (Handy et al., 1961; Parr and Otto, 2013). The maintenance of good general health is important to provide longevity in the work field and good overall quality of life (Moore et al., 2001; Parr and Otto, 2013; Caron-Lormier et al., 2016a). Ophthalmic health and good visual acuity are, especially, important for many working dogs, particularly those whose jobs are visually dominant (e.g., guide dogs for the blind). Maintenance of good visual acuity requires a transparent optical pathway, precise optical refraction, and appropriate neuronal processing in the retina, visual pathways, and visual cortex (Miller and Murphy, 1995). For example, refractive errors can cause an image to appear defocused, leading to poor vision and an overall negative impact on performance (Ofri et al., 2012). However, abnormalities in any of these areas can lead to some degree of visual impairment, even blindness, which ultimately can lead to retirement and even euthanasia of a working dog. The previous surveys of police dogs have shown that ocular disease was responsible for $1.4 \%$ of medical emergencies (Parr and Otto, 2013), 2.4\% of retirement from service (Evans et al., 2007), and 11\% of death and euthanasia (Moore et al., 2001). For guide dogs, eye diseases have been ascribed to constitute $3.2 \%$ of early retirement from service (Caron-Lormier et al., 2016b).

Some breeds that are commonly used as working dogs have a predisposition to heritable or acquired ophthalmic disorders that can cause progressive vision loss that may go unnoticed by the handlers, trainers, or owners; until it, there is moderate-to-advanced visual impairment (e.g., corneal disease, cataracts, and 
progressive retinal atrophy) (Barnett, 1986; Curtis and Barnett, 1989; Kommonen et al., 1997; Kraijer-Huver et al., 2008; Jokinen et al., 2011; Takanosu, 2017). In addition, some working breeds can spontaneously develop refractive errors (myopia or hyperopia) that can have a significant impact on visual acuity (Murphy et al., 1992; Mutti et al., 1999; Soares et al., 2004; Black et al., 2008; Kubai et al., 2008, 2013; Williams et al., 2011). For example, defocus by as little as -1.5 diopters worsens significantly a dog's performance to find distant objects (Murphy et al., 1997; Ofri et al., 2012).

The purpose of this study was to evaluate the prevalence of ophthalmic abnormalities and the refractive states in a population of police and guide dogs in Southern Brazil and to compare the findings with the type of job performed by the dogs and the potential consequences of aberrations in visual quality.

\section{Materials and Methods}

The animals used were service dogs from four different military and police corporations: $20^{\circ}$ Batalhão de Infantaria Blindada $\left(20^{\circ}\right.$ Armored Infantry Battalion-20 $\left.-20^{\circ} \mathrm{BIB}\right)$, Batalhão de Operações Especiais do Paraná (Paraná's Battalion of Special Police Operations-BOPE), Guarda Municipal de Curitiba (Curitiba's Municipal Guard-GMC), and Departamento Estadual de Prevenção e Repressão ao Narcotráfico (State Department for the Prevention and Suppression of Drug Trafficking-DENARC). In addition, the guide dogs used were from the Helen Keller guide dog training school, and there are three privately owned guide dogs. The Comparative Ophthalmology Laboratory of the Federal University of Paraná State conducted this study.

A total of 71 dogs (141 eyes) were evaluated. All dogs underwent a complete eye examination (described below), and 62 who had streak retinoscopy were taken to evaluate their refractive states. Twelve different breeds were presented in this study: American Pit Bull Terrier, Belgian Malinois, Bloodhound, Doberman Pinscher, Dutch Shepherd, Flat-coated Retriever, German Shepherd, Golden Retriever, Labrador Retriever, Rottweiler, and White Swiss shepherd. Belgian Malinois was overrepresented (30 individuals), and a total of $42 \%$ of all dogs were included in the study. All handlers, trainers, and owners responsible for dogs for inclusion in this study granted permission by signed consent. Aggressive dogs were excluded for safety.

\section{History and classification of service tasks}

Gender, age, breed, vaccination, and deworming status were recorded. For each dog, the following was obtained from the owner/handler: 1) description of the service task(s) (e.g., guide dog and narcotic sniffer), 2) their perceived ability of the dog to perform their service task(s), and (3) if any indication of decreased or abnormal vision (e.g., stumbling on obstacles and difference in light vs. dark conditions) has been evident.
Dogs were separated by occupations: police dogs or guide dogs (GD). Police dogs were then subdivided into four different categories: sniffer dogs (narcotics and explosives) (SD), tracking dogs (search and rescue dogs and criminal tracking dogs) (TD), attack and protection dogs (patrol, scout, and sentry dogs) (APD), and other dogs (OD) (agility and dogs remaining early in training with service not yet defined).

\section{Ophthalmic abnormalities}

Schirmer tear test type 1 was performed prior to the administration of any topical medications. Complete ophthalmic evaluations were performed in semidark rooms, both before and after pharmacologic mydriasis with topical tropicamide $1 \%$ (Mydriacyl $®$ and Novartis ${ }^{\circledR}$, São Paulo, Brazil). Ocular reflexes tested consisted of pupillary light reflex, menace response, dazzle reflex, palpebral reflex, and visual tracking using cotton balls. Slit-lamp biomicroscopy (Hawk Eye ${ }^{\circledR}$ and Optotek Medical ${ }^{\circledR}$, Slovenia) and indirect ophthalmoscopy with a Finoff transilluminator (Welch Allyn ${ }^{\circledR}$, New York) and a 20 diopter indirect lens were performed after 10 minutes of tropicamide instillation (Volk Optical Inc. ${ }^{\circledR}$, Mentor, Ohio). Tonometry (Tonovet ${ }^{\circledR}$ and Tiolat Ltd. ${ }^{\circledR}$, Helsinki, Finland) and fluorescein staining (Drogavet $\AA$, Curitiba, Brazil) were also performed.

Ophthalmic abnormalities were classified according to the ocular disorder, which was further categorized as being active or inactive, and whether or not the lesion was located within the visual axis. Those considered active either required clinical or surgical treatment and/ or that could cause a progressive decline in vision or ophthalmic health. The potential to compromise vision due to obstruction of the visual axis was also recorded, and all retinal lesions, despite the location, were considered within the visual axis.

\section{Refractive errors}

Refractive states were assessed to determine the prevalence and severity of refractive errors. Streak retinoscopy was performed using a retinoscope (Welch Allyn ${ }^{\circ}$, New York) and a set of skiascopic lenses (Sunwin $\AA$, China) after pharmacologic mydriasis, 10 minutes after tropicamide instillation. The retinoscope was placed in front of the evaluator's eye with a distance of approximately $65 \mathrm{~cm}$ from the dog. A correction of -1.5 diopters was made due to the distance between the retinoscope and the eye. All the measurements were performed by the same veterinary ophthalmologist (FMF) and required only physical restraint of the animals. Emmetropia was considered when the streak retinoscopy values were between or equal to -0.5 and +0.5 diopters after correction was accounted (Kubai et al., 2008). Ametropia was considered when an eye had myopia (refractive errors were below -0.5 diopters) or hyperopia (when the value was higher than +0.5 diopters). Furthermore, anisometropia was considered when a difference of refractive errors between the eyes of the same dog was $\geq 1.0$ diopters. 


\section{Statistical analysis}

Subjecting the refractive error and age of the animals to the Kolmogorov-Smirnov normality test showed that the distribution was non-Gaussian. Spearman correlations were made for comparison between the eyes of the same dog and for age and refractive errors. Median, interquartile range, and range were calculated for refractive errors and separated by dog service task and breed. The Chi-squared tests were performed to determine if there was a relationship between ocular disease and gender. The Kruskal-Wallis test was used for comparison between the refractive errors of the groups and was adjusted by the Dunn's post hoc method and the Benjamini-Hochberg false discovery rate. The Mann-Whitney test was used to find if the dogs with ophthalmic disorders have a significantly different age compared to those without ophthalmic disorders and to compare the refractive errors between guide dogs and police dogs. A significance was defined if a $p$-value was less than 0.05 . All statistics were analyzed by StatView 5.0 (Cary, NC) for Windows.

\section{Ethical approval}

All aspects of the study were approved by the Animal Use Ethics Committee of the University, protocol number 068/2018.

\section{Results}

History and classification of service tasks

Of the 71 dogs examined, there were more males ( $n$ $=42$, median $2 \pm 2.52$ years old, range: 2 months -9 years) than females $(n=29$, median $1 \pm 1.52$ years old, range: 4 months -10 years). All the age values are presented as median and interquartile range in years. There were a total of 10 guide dogs and 61 police dogs, of which they were separated into 24 sniffers dogs, 10 tracking dogs, 17 attack and protection dogs, and $10 \mathrm{dogs}$ in training or other functions. The details are presented in Table 1. All the dogs were submitted to a rigorous vaccination and deworming programs since their selection was puppies. Altogether, handlers, trainers, and owners said that all the dogs perform their tasks efficiently and they have no perceived evidence of visual impairment.

\section{Ophthalmic abnormalities}

In all, $38(54 \%)$ of the 71 dogs had some ophthalmic abnormality detected, comprising 47 identified abnormalities. The affected dogs were $4 \pm 2.5$ years of age, when compared to $1 \pm 0.17$ years for dogs without abnormalities. The age difference was statistically significant $(p<0.05)$ between the affected and non-

Table 1. Specific ophthalmic abnormalities in working dogs by breed and working group.

\begin{tabular}{|c|c|c|c|c|}
\hline Breed & Group & Total & Affected & Ophthalmic Abnormalities \\
\hline \multirow{4}{*}{ Belgian Malinois } & SD & 18 & 8 & \multirow{4}{*}{$\begin{array}{l}\text { Incipient cataract, chorioretinal scar, vitreous } \\
\text { degeneration, follicular conjunctivitis, pupillary } \\
\text { persistent membrane, enucleated, retinal dysplasia, } \\
\text { corneal dystrophy }\end{array}$} \\
\hline & $\mathrm{TD}$ & 1 & 1 & \\
\hline & APD & 7 & 2 & \\
\hline & OD & 4 & 0 & \\
\hline \multirow{3}{*}{ Labrador Retriever } & GD & 8 & 5 & \multirow{3}{*}{$\begin{array}{l}\text { Pupillary persistent membrane, incipient cataract, } \\
\text { palpebral nodule, nuclear sclerosis, vitreous } \\
\text { degeneration }\end{array}$} \\
\hline & SD & 2 & 2 & \\
\hline & OD & 2 & 2 & \\
\hline \multirow{4}{*}{ German Shepherd } & SD & 2 & 1 & \multirow{4}{*}{$\begin{array}{l}\text { Incipient cataract, dyscoria, distichiasis, nuclear } \\
\text { sclerosis }\end{array}$} \\
\hline & TD & 2 & 1 & \\
\hline & APD & 2 & 2 & \\
\hline & OD & 1 & 0 & \\
\hline Bloodhound & TD & 7 & 6 & Ectropion, incipient cataract, blepharitis, iris atrophy \\
\hline \multirow{2}{*}{ Rottweiler } & APD & 5 & 3 & \multirow{2}{*}{$\begin{array}{l}\text { Incipient cataract, chorioretinal scar, pupillary persistent } \\
\text { membrane }\end{array}$} \\
\hline & OD & 1 & 1 & \\
\hline Golden Retriever & GD & 1 & 1 & Distichiasis \\
\hline Flat-coated Retriever & GD & 1 & 1 & Follicular conjunctivitis \\
\hline Dutch Shepherd & SD & 2 & 1 & Ectropion uveae \\
\hline Doberman Pinscher & APD & 2 & 1 & Incipient cataract, iris atrophy \\
\hline American Pit Bull Terrier & APD & 1 & 0 & N/A \\
\hline \multirow[t]{2}{*}{ White Swiss Shepherd } & OD & 2 & 0 & N/A \\
\hline & Total & 71 & 38 & \\
\hline
\end{tabular}

(GD): Guide dogs; (SD): sniffer dogs; (TD): tracking dogs; (APD): attack and protection dogs; (OD): other dogs; (N/A): no abnormalities detected. 
affected dogs. There was no significant difference in gender $(p>0.05)$.

The most prevalent ophthalmic abnormality was incipient cataracts, affecting $15(21 \%)$ dogs $(5 \pm 1.5$ years). Twelve cataracts were incipient, occupying less than $5 \%$ of the lens diameter, and were out of the visual axis, and three were within the visual axis (two within the lens nucleus and one on the anterior lens capsule). The affected breeds were Belgian Malinois (5), Labrador retriever (3), German Shepherd (3), Bloodhound (2), Doberman Pinscher (1), and Rottweiler (1).

The second most prevalent abnormality was persistent pupillary membranes, affecting five dogs. One dog had the right eye removed due to trauma in the early months of age. Other identified ophthalmic abnormalities included nuclear sclerosis (4), chorioretinal scars (3), vitreous degeneration (3), follicular conjunctivitis (2), ectropion (2), blepharitis (2), distichiasis (2), iris atrophy (2), ectropion uveae (2), corneal dystrophy (1), retinal dysplasia (1), palpebral nodule (1), and dyscoria (1). Of these abnormalities, 28 (60\%) of them were considered potentially inherited forms of cortical incipient cataracts, persistent pupillary membranes, vitreous degeneration, ectropion, distichiasis, corneal dystrophy, and retinal dysplasia.

Considering the location of the abnormalities, 10 dogs had an abnormality within the visual axis, of which four were chorioretinal scars, three nuclear sclerosis, one dog with pupillary persistent membrane and cataract, and two dogs with nuclear incipient cataract (one of which also had nuclear sclerosis). Dogs with abnormalities within the visual axis included SD (5), TD (1), and APD (4). None of GD and OD group had ophthalmic abnormality on the visual axis. A total of 25 detected ophthalmic abnormalities in 24 dogs were considered active: cataracts (15), follicular conjunctivitis (2), blepharitis (2), distichiasis (2), retinal dysplasia (1), ectropion (2), and a palpebral nodule (1). Dogs with abnormalities considered to be active included GD (3), SD (7), TD (7), APD (6), and OD (1). Only five dogs had ophthalmic abnormalities that were considered to be active and were within the visual axis. The details of the distribution of ophthalmic abnormalities are presented in Table 1. Photographs of dogs with ophthalmic abnormalities are shown in Figure 1.

\section{Refractive errors}

Concerning refractive errors of the 62 dogs submitted to streak retinoscopy, 123 eyes were evaluated. Of the 62 dogs (123 eyes), the median and interquartile range of refractive errors was $-0.75 \pm 0.75$ for all the eyes, $-1 \pm 0.72$ for the left eyes, and $-0.75 \pm 0.94$ for the right eyes. The correlation coefficient between the eyes of the same dog was $\mathrm{R}=0.94$ and was statistically significant $(p<0.05)$. The correlation coefficient between refractive error and age was $\mathrm{R}=0.10$ and was not significant $(p>0.5)$. Of the 123 eyes, only eight were found to have no refractive error (0 diopters) and only two dogs were found to have no refractive error in both the eyes. Of the 62 dogs, only $12(19 \%)$ were considered emmetropes, in which five of them were Labrador Retrievers. The other 50 dogs were ametropic (81\%), of them $40(65 \%)$ were myopics and $10(16 \%)$ hyperopics. Only three dogs $(0.05 \%)$ were anisometropic (two Belgian Malinois and one Labrador Retriever), with a difference of 1.0 diopter between the eyes. A histogram graph of the distribution of refractive error is shown in Figure 2. The details of refractive errors and the dog breed independent on their function are presented in Table 2 .

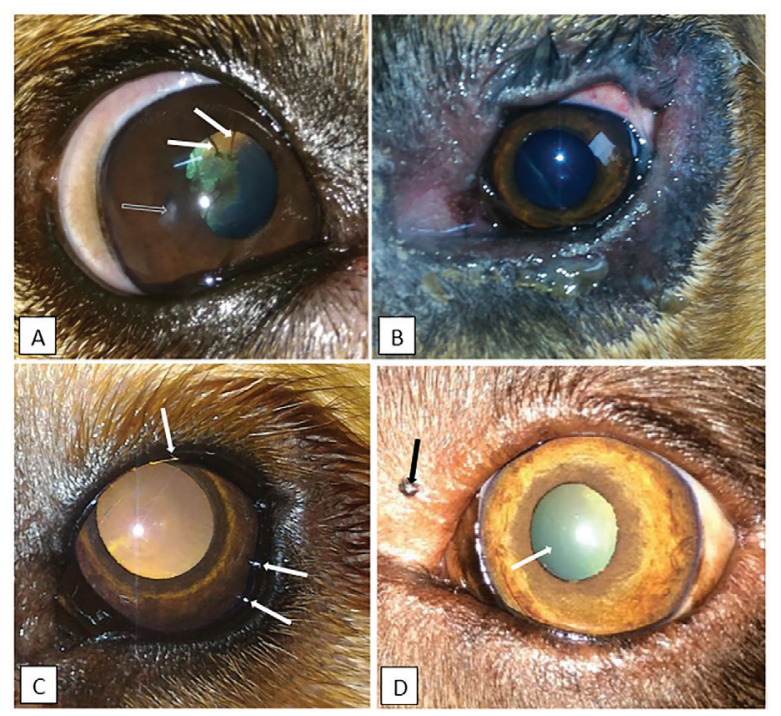

Fig. 1. Photographs of some of the ophthalmic abnormalities found in working dogs. A: Pupillary persistent membrane (white arrow) and corneal opacity (black arrow) in a 4-yearold male Belgian Malinois. B: blepharitis in a 1-year-old female Bloodhound. C: distichiasis (white arrows) in a 1-year-old female Golden Retriever. D: pigmented palpebral nodule (black arrow) and nuclear sclerosis (white arrow) in an 8-year-old female Labrador Retriever.

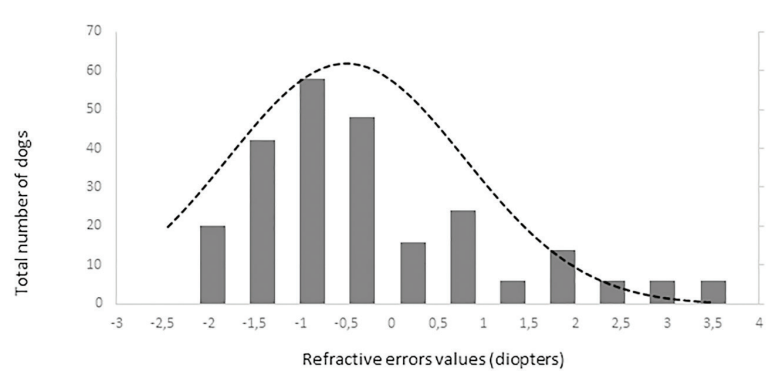

Fig. 2. Histogram graph of the distribution of refractive errors of 62 police and guide dogs. Note the skewed distribution, showing the tendency toward myopia. The dashed line represents the distribution of refractive errors. 
A significant difference $(p<0.05)$ was detected between the refractive state of police dogs $(-1.0 \pm 0.5)$ and guide dogs $(0.25 \pm 0.75)(p<0.05)$. A comparison between $\mathrm{SD}, \mathrm{APD}, \mathrm{TD}$, and OD showed no significant statistical differences $(p>0.05)$. GD was significantly different from all the groups $(p<0.05)$ with exception of TD $(p=0.52)$. The details of dog function and refractive errors are shown in Table 3, and the distribution among the groups is shown on a box plot graph in Figure 3 .

\section{Discussion}

Ophthalmic abnormalities and ametropia were present in most of the working dogs studied. Older dogs had more ophthalmic abnormalities than younger dogs, and inherited ophthalmic abnormalities were the most common type found. Incipient cortical cataract and pupillary persistent membrane were the most prevalent abnormality detected. Despite the high frequency of ophthalmic abnormalities, few were located in the

Table 2. Refractive errors (RE) of working dogs by breed, displayed as median \pm interquartile range $(M \pm I R)$ and range $(R)$.

\begin{tabular}{lccc}
\hline Breed & \# of Dogs & RE $(\mathbf{M} \pm \mathbf{I R})$ & RE $(\mathbf{R})$ \\
\hline Belgian Malinois & 24 & $-1.00 \pm 0.69$ & $-2.25 ;+3.50$ \\
Labrador Retriever & 12 & $+0.50 \pm 0.72$ & $-2.50 ;+2.50$ \\
German Shepherd & 7 & $-1.25 \pm 0.34$ & $-2.50 ; 0$ \\
\hline Bloodhound & 7 & $+0.50 \pm 0.94$ & $-1.25 ;+1.50$ \\
Rottweiler & 6 & $-1.13 \pm 0.38$ & $-2.25 ;-0.75$ \\
Doberman Pinscher & 2 & $-1.00 \pm 0.25$ & $-1.25 ;-0.75$ \\
Swiss White Shepherd & 2 & $-1.50 \pm 0.34$ & $-2.0 ;-0.75$ \\
Flat-coated Retriever & 1 & $-2.13 \pm 0.06$ & $-2.25 ;-2.0$ \\
Golden Retriever & 1 & $-0.86 \pm 0.06$ & $-1.0 ;-0.75$ \\
American Pit Bull Terrier & 1 & $-1.5 \pm 0$ & $-1.5 ;-1.5$ \\
\hline
\end{tabular}

Table 3. Refractive errors (RE) in working dogs by breed (median of right, left, and both eyes) and by working group (median \pm interquartile range).

\begin{tabular}{|c|c|c|c|c|c|}
\hline Group & Breed & RE (Right) & RE (Left) & RE (Both) & RE (Both) \\
\hline \multirow{3}{*}{ Guide dogs } & Labrador Retriever & +0.5 & +0.13 & +0.5 & \multirow{3}{*}{$0.38 \pm 0.71$} \\
\hline & Golden Retriever & -0.75 & -1 & -0.86 & \\
\hline & Flat-coated Retriever & -2 & -2.25 & -2.13 & \\
\hline \multirow{3}{*}{ Sniffer dogs } & Belgian Malinois & -0.86 & -1 & -1 & \multirow{3}{*}{$-0.75 \pm 0.75$} \\
\hline & German Shepherd & -0.5 & -0.75 & -0.75 & \\
\hline & Labrador Retriever & -0.38 & -0.63 & -0.38 & \\
\hline \multirow{3}{*}{ Tracking dogs } & Bloodhound & +0.5 & +0.5 & +0.5 & \multirow{3}{*}{$-0.50 \pm 1.13$} \\
\hline & German Shepherd & -1.38 & -1.38 & -1.38 & \\
\hline & Belgian Malinois & -1.5 & -1.75 & -1.63 & \\
\hline \multirow{5}{*}{ Attack and protection dogs } & Belgian Malinois & -0.63 & -0.75 & -0.63 & \multirow{5}{*}{$-1.25 \pm 0.38$} \\
\hline & Rottweiler & -1.5 & -1.25 & -1.38 & \\
\hline & German Shepherd & -2 & -1.13 & -1.87 & \\
\hline & Doberman Pinscher & -1 & -1 & -1 & \\
\hline & American Pit Bull Terrier & -1.5 & -1.5 & -1.5 & \\
\hline \multirow{5}{*}{ Others } & Belgian Malinois & -0.63 & -0.38 & -0.38 & \multirow{5}{*}{$-1.13 \pm 0.41$} \\
\hline & White Swiss Shepherd & -1.25 & -1.63 & -1.5 & \\
\hline & Labrador Retriever & -0.13 & -0.13 & -0.13 & \\
\hline & German Shepherd & -1.25 & -1.25 & -1.25 & \\
\hline & Rottweiler & -1 & -1 & -1 & \\
\hline
\end{tabular}




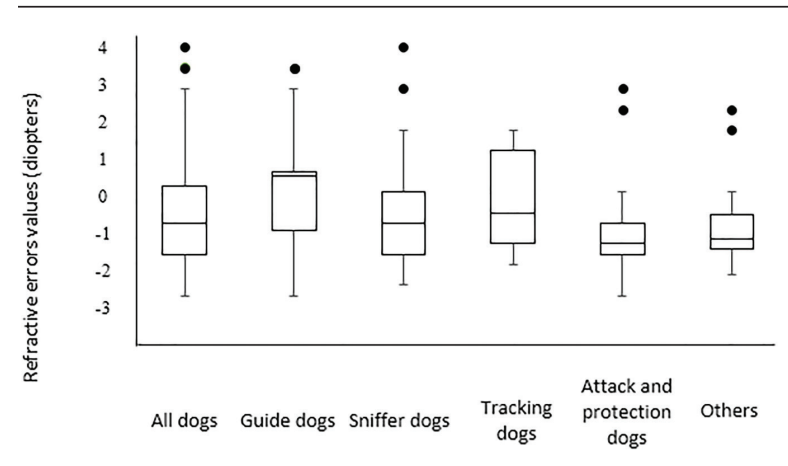

Fig. 3. Box plot graph of the refractive errors of 62 police and guide dogs. All dogs are displayed in the first column and subsequently divided into five groups. Both "attack and protection dogs" and "others" have narrower ranges when compared to other groups.

visual axis and even fewer located outside of the visual axis would likely significantly impair vision. Overall, working dogs tended to be myopic although GD and TD tended to be emmetropic. Although ophthalmic abnormalities or ametropia were seen in the majority of the dogs, abnormalities were mostly very minor, and working performance was good with no reports of visual impairment noted by any handlers, trainers, or owners.

Only 10 ophthalmic abnormalities were located within the visual axis and were only found in police dogs. TD and SD rely more on olfaction rather than vision (Gazit and Terkel, 2003), and thus, the ocular abnormalities found probably do not significantly impact their performance. Interestingly, one SD who had a unilateral enucleation yet does not have an appreciable decline in performance. APD may rely on vision more than TD and $\mathrm{SD}$ as a means to intercept potential criminals; however, ophthalmic abnormalities within the visual axis were detected in this group. A decline in visual acuity has been hypothesized as a contributing factor in increased aggressiveness (Helms, 2008), which can be a concern for maintaining control of the dog by the trainer and handler. However, no complaints about the visual performance of the affected APDs were noted, and thus, small changes in visual acuity may not have a detrimental impact on the ability of APD to perform well.

Similarly, police dogs were found to largely be ametropic, all groups averaging $-0.75-1.25$ diopters myopic, similar to some studies of pet dogs and Brazilian police dogs (Helms, 2008; Groth et al., 2013). Rottweilers, German Shepherds, and Belgian Malinois have been shown to commonly have myopia (Murphy et al., 1992; Helms, 2008), and all were common breeds in the SD, APD, and OD groups in the present study. Other reports from Brazil have shown a tendency for dogs to be hyperopic in a limited survey of 10 animals and in a population of Brazilian mastiffs (Soares et al., 2004; Mobricci, 2006; Ranzani et al., 2008), whereas emmetropia has been described in the majority of dogs (Kubai et al., 2008; 2013; Williams et al., 2011).
The working dogs can identify moving objects at a distance of $810-900 \mathrm{~m}$ and stationary objects at $585 \mathrm{~m}$ (Walls, 1963). However, with myopia of at least -2.0 diopters, the reduction in visual acuity can negatively influence their performance and limit object identification to less than 140 m (Murphy et al., 1997; Ofri et al., 2012). However, the trainers, handlers, or owners reported no evidence of poor visual acuity or low performance. This could be not only due to the low degrees of myopia found in the majority of dogs but also that working dogs in the police groups who could rely their perception more on other senses to successfully perform their jobs, and thus, low myopia may not have a significant impact of working performance in these groups (Gazit and Terkel, 2003).

Interestingly, one TD breed, the Bloodhound, was found to be emmetropic $(+0.5$ diopters). Some individuals had slight hyperopia, similar to the findings in Brazilian mastiffs from a previous study (Soares et al., 2004), which are similar breeds. Further investigation into the tendency toward hyperopia in bloodhounds and related breeds to determine if there exists a predisposition and the possibility of a naturally occurring animal model for hyperopia is recommended.

$\mathrm{GD}$, on the other hand, relies heavily on vision for performance success. We found that GD had no ophthalmic abnormalities within the visual axis and had a tendency toward emmetropia, similar to what has been described in the previous studies (Murphy et al., 1992; Kubai et al., 2008). Selection for GDs is largely based on the potential to excel in visually dominant tasks, such as the ability to safely lead a visually impaired person. During the first month of age, potential GDs are evaluated for behavior, quality of interactions, and trainability, and the time to train a dog with good visual acuity is faster than the one with poor visual acuity (Kubai et al., 2008). Due to direct selection based on performance, having low refractive errors could be a heritable trait breed into working lines (Murphy et al., 1992; Kubai et al., 2008). The previous studies have shown significant differences in refractive errors between guide dogs and nonworking dogs of the same breed, with guide dogs tending to be more emmetropic than nonworking dogs (Kubai et al., 2008). Labrador Retrievers have a naturally occurring myopia due to the lengthening of vitreous chamber (Mutti et al., 1999; Kubai et al., 2008). However, this breed was largely emmetropic in the present study, as found in some previous surveys (Mutti et al., 1999; Black et al., 2008). Most of the Labrador Retrievers in the present study belonged to the GD group, who are critically dependent and selected for good visual acuity. Another example was shown in a German Shepherd population of guide dogs, another breed predisposed to myopia, where the investigators found that a majority of the dogs were emmetropic (Murphy et al., 1992), suggesting that the guide dog population may, overall, have better visual acuity. 
Some of the ocular diseases diagnosed in this study are presumably inherited, especially, considering that all dogs were purebred (Barnett, 1978; Kraijer-Huver et al., 2008; ACVO, 2015). Cataracts were seen in 21\% of dogs, similar to some previous surveys ( $14.7 \%$ to 31.4\%) (Donzel et al., 2016; Guandalini et al., 2017), although more than three times higher than other investigations looking at specific breeds (Curtis and Barnett, 1989; Gelatt and Mackay, 2005; KraijerHuver et al., 2008; Urfer et al., 2011). Cataracts can be heritable in purebred, which may be a reason for the high prevalence found in the present study. In some areas, the incidence of heritable cataracts has been increasing with time (Gelatt and Mackay, 2005), possibly due to the absence of registration and controlled breeding of affected dogs with suspected heritable eye disease.

Labrador Retrievers are predisposed to several ophthalmic conditions that can cause blindness, such as cataracts and progressive retinal atrophy (PRA) (Curtis and Barnett, 1989; Kommonen et al., 1997; PetersenJones, 1998; Gelatt and Mackay, 2005; Kraijer-Huver et al., 2008), and the latter being the most common ocular disease causing early retirement of guide dogs (Caron-Lormier et al., 2016a). PRA has an autosomal recessive mode of inheritance in this breed, and the gene mutation is more prevalent in pet dogs than guide dogs, likely due to the rigorous selection process of guide dogs (Takanosu, 2017). No Labrador Retrievers in the present study had detectable PRA. However, three of them had incipient cortical cataracts at the posterior pole, which are inherited in this breed (Curtis and Barnett, 1989; Kraijer-Huver et al., 2008). This type of cataract can be progressive, so future ophthalmic evaluations are important.

Fundus abnormalities, such as chorioretinal scars, in police dogs were detected at similar rates as a previous report on German Shepherd police dogs (Balicki et al., 2013). Good visual performance can be maintained with small, static chorioretinal lesions not located at the area centralis (Balicki et al., 2013). The cause of the chorioretinal scars is unclear; however, intense physical activity or stress causing elevations in blood pressure could lead to microvascular hemorrhages and/or focal retinal detachment and thus scarring of the retina (Storey et al., 2005; Balicki et al., 2013). Other causes cannot be excluded, such as inactive chorioretinitis caused by infectious diseases and intraocular larva migrans caused by Toxocara $s p$. that lead to granulomatosis of the retina and choroid in dogs (Rubin and Saunders, 1965); the second was found to be a potential cause of chorioretinal lesions in sheep dogs, another category of working dogs (Hughes et al., 1987). Even the vaccination and deworming status of the dogs in this study were up to date, these diseases are an important differential diagnosis. Further, when chorioretinal lesions are found in working dogs, they need to be investigated due to its potential to cause blindness.
Ophthalmic abnormalities were more common in older dogs, who are more frequently withdrawn from service due to ocular disorders than younger dogs (Evans et al., 2007). The increased number of abnormalities in older dogs suggests late onset and potentially progression with age, particularly nuclear sclerosis and senile cataracts (Williams et al., 2004; Gelatt and Mackay, 2005; Urfer et al., 2011). In large breeds, the onset of senile cataracts occurs after 6 years of age with an increasing prevalence in age (Riederle, 1999) although senile cataracts often do not reach the mature stage (Williams et al., 2004). However, we did not find a correlation between age and refractive error, probably due to the low number of older dogs developing nuclear sclerosis, which can shift the refractive state toward myopia (Murphy et al., 1992; Kubai et al., 2008; Maehara et al., 2011).

\section{Conclusions}

Ophthalmic abnormalities and refractive errors were not uncommon within a population of working dogs in South Brazil. However, no evidence of significant vision loss or decreased work performance was noted. Police dogs have a tendency for myopia, whereas guide dogs were emmetropic. Ophthalmic evaluations should be performed regularly in working dogs to maintain eye health and visual acuity. In addition, some of these ocular abnormalities can progress, and the follow-up is essential. Many of these findings are genetically inheritable, and thus, the removal of affected dogs and their parents from breeding is recommended.

\section{Acknowledgments}

The authors would like to thank the following institutions: $20^{\circ} \mathrm{BIB}, \mathrm{BOPE}, \mathrm{GMC}$, DENARC, Helen Keller guide dog school, and guide dog owners which made this study possible.

\section{Conflict of interest}

The author(s) declared no potential conflicts of interest with respect to the research, authorship, and/or publication of this article.

\section{Authors' contributions}

FMF and JKO performed all the ophthalmic examinations on working dogs and wrote the manuscript. MB, MS, and RKS helped handling the dogs and with data collection. BAM helped with the manuscript writing and statistical analyses.

\section{References}

American College of Veterinary Ophthalmologists (ACVO). 2017. The blue book. Ocular disorders presumed to be inherited in purebred dogs, 10th ed. Available via www.ofa.org > uploads > 2018/09 > bluebook-public-092518 (Assessed December 2019).

Balicki, I., Nestorowicz, N. and Ofri, R. 2013. Funduscopic abnormalities and electroretinography 
in cases of retinopathy in German Shepherd dogs. Vet. Ophthalmol. 16, 397-408.

Barnett, K.C. 1986. Hereditary cataract in the German Shepherd dogs. J. Small Anim. Pract. 27, 387-395.

Barnett, K.C. 1978. Hereditary cataract in the dog. J. Small Anim. Pract. 19, 109-120.

Black, J., Browning, S., Collins, A.V. and Phillips, J.R. 2008. A canine model of inherited myopia: familial aggregation of refractive error in Labrador Retrievers. Invest. Ophthalmol. Vis. Sci. 49, 47844789.

Caron-Lormier, G., England, G.C.W., Green, M.J. and Asher, L. 2016a. Using the incidence and impact of health conditions in guide dogs to investigate healthy ageing. Vet. J. 207, 124-130.

Caron-Lormier, G., Harvey, N.D., England, G.C.W. and Asher, L. 2016b. A new metric for quantifying the relative impact of risk factors on loss of working life illustrated in a population of working dogs. PLoS One. 11, e0165414.

Craigon, P.J., Hobson-West, P., England, G.C.W., Whelan, C., Lethbridge, E. and Asher, L. 2017. "She's a dog at the end of the day": Guide dog owner's perspectives on the behavior of their guide dog. PLoS One. 12, e0176018.

Curtis, R. and Barnett, K.C. 1989. A survey of cataracts in Golden and Labrador Retrievers. J. Small Anim. Pract. 30, 277-286.

Dever, S. 2015. Dog and shield: the buffering effect K-9s have on Police Officer Strain. Honors Thesis. Salem, MA: Salem State Univ.

Donzel, E., Arti, L. and Chahory, S. 2016. Epidemiology and clinical presentation of canine cataracts in France a retrospective study of 404 cases. Vet. Ophthalmol. 20, 131-139.

Evans, R.I., Herbold, J.R., Bradshaw, B.S. and Moore, G.E. 2007. Causes for discharge of military working dogs from service: 268 cases (2000-2004). J. Am. Vet. Med. Assoc. 231, 1215-1220.

Fishman, G.A. 2003. When your eyes have a wet nose: the evolution of the use of guide dogs and establishing the seeing eye. Surv. Ophthalmol. 48, 452-458.

Gazit, I. and Terkel, J. 2003. Domination of olfaction over vision in explosives detection by dogs. Appl. Anim. Behav. Sci. 82, 65-73.

Gelatt, K.N. and Mackay, E.O. 2005. Prevalence of primary breed-related cataracts in the dog in North America. Vet. Ophthalmol. 8, 101-111.

Groth, A.D., Hollingsworth, S.R., Ofri, R., Kass, P.H., Reed, Z. and Murphy, C.J. 2013. Clinical comparison of the Welch Allyn SureSight ${ }^{\mathrm{TM}}$ handheld autorefractor versus streak retinoscopy in dogs. Vet. Ophthalmol. 16, 319-323.

Guandalini, A., Girolamo, N.D., Corvi, R., Santillo, D., Andreani, V. and Pinzo, B. 2017 Epidemiology of ocular disorders presumed to be inherited in three small Italian dog breeds in Italy. Vet. Ophthalmol. 21, 524-529.

Handy, W., Harrington, M. and Pittman, DJ. 1961. The K-9 Corps: the use of dogs in Police work. J. Crim. L. Criminol. 52, 328-337.

Helms, G. 2008. Retinoscopia em cães de trabalho militar: correlação entre ametropias e alterações comportamentais. M.S. Thesis, Universidade Federal Fluminense, Niterói, Brazil.

Hughes, P.L., Dubielzig, R. and Kazacos, K.R. 1987. Multifocal retinitis in New Zealand sheep dogs. Vet. Pathol. 24, 22-27.

Jokinen, P., Rusanen, E.M., Kennedy, L.J. and Lohi, H. 2011. MHC class II risk haplotype associated with Canine chronic superficial keratitis in German shepherd dogs. Vet. Immunol. Immunopathol. 140, $37-41$.

Kommonen, B., Kylmä, T., Karhunen, U., Dawson, W.W. and Penn, J.S. 1997. Impaired retinal function in young Labrador retriever dogs heterozygous for late onset rod-cone degeneration. Vision Res. 37, 365-370.

Kraijer-Huver, I.M.G., Gubbels, E.J., Scholten, J., Djajadiningrat-Laanen, S.C., Boevé, M.H. and Stades, F.C. 2008. Characterization and prevalence of cataracts in Labrador Retrievers in The Netherlands. Am. J. Vet. Res. 69, 1336-1340.

Kubai, M.A., Labelle, A.L., Hamor, R.E., Mutti, D.O., Famula, T.R. and Murphy, C.J. 2013. Heritability of lenticular myopia in English Springer Spaniels. Invest. Ophthalmol. Vis. Sci. 54, 7324-7328.

Kubai, M.A., Bentley, E., Miller, P.E., Mutti, D.O. and Murphy, C.J. 2008. Refractive states of eyes and association between ametropia and breed in dogs. Am. J. Vet. Res. 69, 946-951.

Maehara, S., Itoh, Y., Higashonozono, K. and Izumisawa, Y. 2011. Evaluation of refractive value by skiascopy in healthy beagles. J. Vet. Med. Sci. 73, 927-929.

Miller, P.E. and Murphy, C.J. 1995. Vision in dogs. J. Am. Vet. Med. Assoc. 207(12), 1623-1634.

Mobricci, L.A.L. 2006. Avaliação do erro refracional por retinoscopia com luz em faixa em cães fácicos, afácicos e pseudofácicos. Dr. Sci. Thesis, Faculdade de Medicina Veterinária e Zootecnia. Universidade Estadual Paulista. Botucatu, Brazil.

Moore, G.E., Burkman, K.D., Carter, M. and Peterson, M.R. 2001. Causes of death or reasons for euthanasia in military working dogs: 927 cases (1993-1996). J. Am. Vet. Med. Assoc. 219, 209-214.

Murphy, C.J., Mutti, D.O., Zadnik, K. and Hoeve, J.V. 1997. Effect of optical defocus on visual acuity in dogs. Am. J. Vet. Res. 58, 414-418.

Murphy, C.J., Zadnik, K. and Mannis, M.J. 1992. Myopia and refractive error in dogs. Invest. Ophthalmol. Vis. Sci. 33, 2459-2463.

Mutti, D.O., Zadnik, K. and Murphy, C.J. 1999. Naturally occurring vitreous chamber-based myopia 
in the Labrador retriever. Invest. Ophthalmol. Vis. Sci. 40, 1577-1584.

Ofri, R., Hollingsworth. S.R., Groth, A., Motta, M.J., Doval, J.H., Kass, P.H. and Murphy, C.J. 2012. Effect of optical defocus on performance of dogs involved in field trial competition. Am. J. Vet. Res. 73, 546-550.

Parr, J.R. and Otto, C.M. 2013. Emergency visits and occupational hazards in German shepherd police dogs (2008-2010). J. Vet. Emerg. Crit. Care 23, 591-597.

Petersen-Jones, S.M. 1998. A review of research to elucidate the causes of the generalized progressive retinal atrophies. Vet. J. 155, 5-18.

Ranzani, J.J.T., Mobricci, L.A.L., Carvalho, L.R. and Brandão, C.V.S. 2008. Refração por retinoscopia com luz em faixa em cães. Arq. Bras. Med. Vet. Zootec. 60, 353-355.

Riederle, G. 1999. The importance of the guide dog. Does the guide dog still have a place in the next century? Rehabilitation (Stuttg) 38, 33-37.

Rubin, L.F. and Saunders, L.Z. 1965. Intraocular larva migrans in dogs. Pathol. Vet. 2, 566-573.

Sloane, C.F. 1955. Dogs in war, police work and on patrol. J. Crim. Law Criminol. 46, 385-395.
Soares, A.M.B., Laus, J.L., Provenzano, J., Ayeres, B., Marssilac, P.D., Millward, C. and Galera, P.D. 2004. Ocular refraction by streak retinoscopy in the canine Fila Brasileiro breed. Revista Brasileira de Ciência Veterinária 11, 104-108.

Storey, E.S., Grahn, B.H. and Alcorn, J. 2005. Multifocal chorioretinal lesions in Borzoi dogs. Vet. Ophthalmol. 8, 337-347.

Takanosu, M. 2017. Different allelic frequency of progressive rod-cone degeneration in two populations of Labrador Retrievers in Japan. J. Vet. Med. Sci. 79, 1746-1748.

Urfer, S.R., Greer, K. and Wolf, N.S. 2011. Age-related cataract in dogs: a biomarker for life span and its relation to body size. Age 33, 451-460.

Walls, G.L. 1963. Adaptations to space and motion In The vertebrate eye and its adaptive radiation. Eds., Walls, G.L. New York: Hafner Publishing Co.

Williams, L.A., Kubai, M.A., Murphy, C.J. and Mutti, D.O. 2011. Ocular components in three breeds of dogs with high prevalence of myopia. Optom. Vis. Sci. 88, 269-274.

Williams, D.L., Heath, M.F. and Wallis, C. 2004. Prevalence of canine cataract: preliminary results of a cross-sectional study. Vet. Ophthalmol. 7, 29-35. 